\title{
Hodgkin Lymphoma in a Case of Chronic Myeloid Leukemia Treated with Tyrosine Kinase Inhibitors
}

\author{
Smeeta GAJENDRA, Archana SHARMA, Rashi SHARMA, Sunil Kumar GUPTA, Nitin SOOD, Ritesh SACHDEV
}

Department of Pathology \& Laboratory Medicine, Medanta the Medicity, GURGAON, INDIA

\begin{abstract}
Chronic myeloid leukemia (CML) is characterized by increased and unregulated proliferation of granulocytic lineage in the bone marrow and presence of these immature myeloid cells in the peripheral blood with presence of Philadelphia (Ph) chromosome. Tyrosine kinase inhibitors are the most important drugs in the CML therapy and provide long disease-free survival. Due to the increased survival of CML patients with continual administration of these drugs, the chance of development of secondary malignancies may increase. The most common secondary malignancies are prostate, colorectal and lung cancer, non-Hodgkin lymphoma, malignant melanoma, non-melanoma skin tumors and breast cancer. Herein, we are describing a rare case of Hodgkin lymphoma in a patient of CML after ten year of primary disease presentation. Hodgkin lymphoma in a known case of CML is very rare and further studies are also needed to know the pathogenic relationship between the two entities and to assess the risk of secondary Hodgkin lymphoma in CML patients treated with tyrosine kinase inhibitors. CML itself is a risk factor for development of solid cancers and hematologic malignancies. In addition, patients on chemotherapy are immune-compromised and may be at greater risk of neoplasm driven by infectious agents such as Epstein-Barr virus.
\end{abstract}

Key Words: Hodgkin lymphoma, Imatinib, Chronic myeloid leukemia

\section{INTRODUCTION}

Chronic myeloid leukemia (CML) is characterized by increased and unregulated proliferation of granulocytic lineage in the bone marrow and presence of these immature myeloid cells in the peripheral blood. The cytogenetic marker of CML is Philadelphia $(\mathrm{Ph})$ chromosome (present in granulocytes, monocyte-macrophages, erythroblasts, megakaryocytes, B-lymphocytes) generated by translocation between the long arm of chromosome 9 and 22 designated as $\mathrm{t}(9 ; 22)(\mathrm{q} 34 ; \mathrm{q} 11)(1)$. Various therapeutic approaches for CML patients are available as tyrosine kinase inhibitors (TKI) which target against a set of tyrosine kinase proteins: first generation TKI (Imatinib mesylate) and second generation TKI (in case of Imatinib resistance: Nilotinib, Dasatinib, Bosutinib), allogeneic stem cell transplantation and other therapeutic options such as Interferon alpha (2). These agents have a remarkable efficacy in treating CML patients and provide long diseasefree survival. Due to the increased survival of these patients with continual administration of these drugs, the chance of development of secondary malignancies may increase. An increased incidence of second neoplasms of stomach, skin, urogenital tract as well as lymphoid leukemias has been documented in the literature (3). Herein, we are describing a rare case of Hodgkin lymphoma in a patient of CML ten years after primary disease presentation.

(Turk Patoloji Derg 2019, 35:74-78)

Received : 21.03.2016 Accepted : 23.06.2016

\section{CASE REPORT}

A 50-year-old male had been diagnosed as CML 10 years previously. The peripheral blood smear showed hyperleucocytosis with neutrophil and myelocyte peak and basophilia (Figure 1A). The Quantitative Real time polymerase chain reaction (RT-PCR) for BCR-ABL was $91.0 \%$ at the time of diagnosis. He was started on Imatinib $400 \mathrm{mg}$ daily. After one month of therapy, he achieved complete hematologic response. Cytogenetic remission was noted at the 6th month of the therapy. In December 2013, he stopped chemotherapy till April 2014 when again he was started on Imatinib. In August 2014, the BCRABL was found to be $27.38 \%$. Chromosomal analysis revealed 46XY; $\mathrm{t}(9 ; 10 ; 22)(\mathrm{q} 34 ; \mathrm{p} 11.2 ; \mathrm{q} 11.2)(11) / 46, \mathrm{XY}$ (9). Mutation analysis showed E255V mutation in $100 \%$ of cells. In view of the additional chromosomal abnormalities and E255V mutation, he was started on dasatinib. In March 2015 , he presented to our institution with the complaint of fever. Contrast enhanced Computed Tomography (CECT) of chest showed right-sided pleural effusion with mediastinal and hilar lymphadenopathies. CECT abdomen showed para-aortic, aorto-caval and retro-caval lymphadenopathies. Bronchoscopy was performed and the cytology was negative for malignancy or granulomas. The patient was started on anti-tubercular drugs for 2 months on clinical suspicion with no improvement

Correspondence: Smeeta GAJENDRA

Medanta the Medicity, Department of Pathology \& Laboratory Medicine, GURGAON, INDIA

E-mail: drsmeeta@gmail.com Phone: +09013590875 
in symptoms. Positron emission tomography (PET)/ CECT scan showed enlarged hypermetabolic cervical, mediastinal, hilar, mesenteric, retroperitoneal and abdomino-pelvic lymphadenopathies above and below the diaphragm with hypermetabolic splenic involvement. Complete blood count showed hemoglobin of $123 \mathrm{~g} / \mathrm{L}$, total leucocyte count of $3.73 \times 10^{9} / \mathrm{L}$, and platelets of $330 \times 10^{9} / \mathrm{L}$. Peripheral blood smear showed no abnormal cells. BCRABL (RT-PCR) was $0.0 \%$. Supraclavicular lymph node biopsy showed atypical polymorphous infiltrate composed of scattered large multilobulated Reed-Sternberg (RS) cells in a reactive background which consists of lymphocytes, plasma cells, histiocytes and eosinophils (Figure 1B). On immunohistochemistry, these large cells were positive for CD30 (Figure 1C), CD15 (Figure 1D), MUM-1, PAX5 (weak variable) and negative for MPO, leucocyte common antigen (CD45), CD3 and CD20; suggesting a diagnosis of Classical Hodgkin Lymphoma. Fluorescence in situ hybridisation method to detect Epstein-Barr virus (EBV) DNA was negative. Bone marrow examination done for staging showed infiltration by Reed-Sternberg cells (Figure $1 \mathrm{E}$ ) which were $\mathrm{CD} 30$ positive (Figure $1 \mathrm{~F}$ ), compatible with involvement by Hodgkin lymphoma. A final diagnosis of stage IV Hodgkin lymphoma in a case of CML was made. The patient was scheduled for ABVD (adriamycin, bleomycin, vinblastine, dacarbazine)-based chemotherapy for 6 cycles. After completion of 3 cycles of chemotherapy, PET-CT was performed and revealed complete metabolic and significant morphological regression of all the previous nodal and extra-nodal disease, with no new metabolically active lesion.
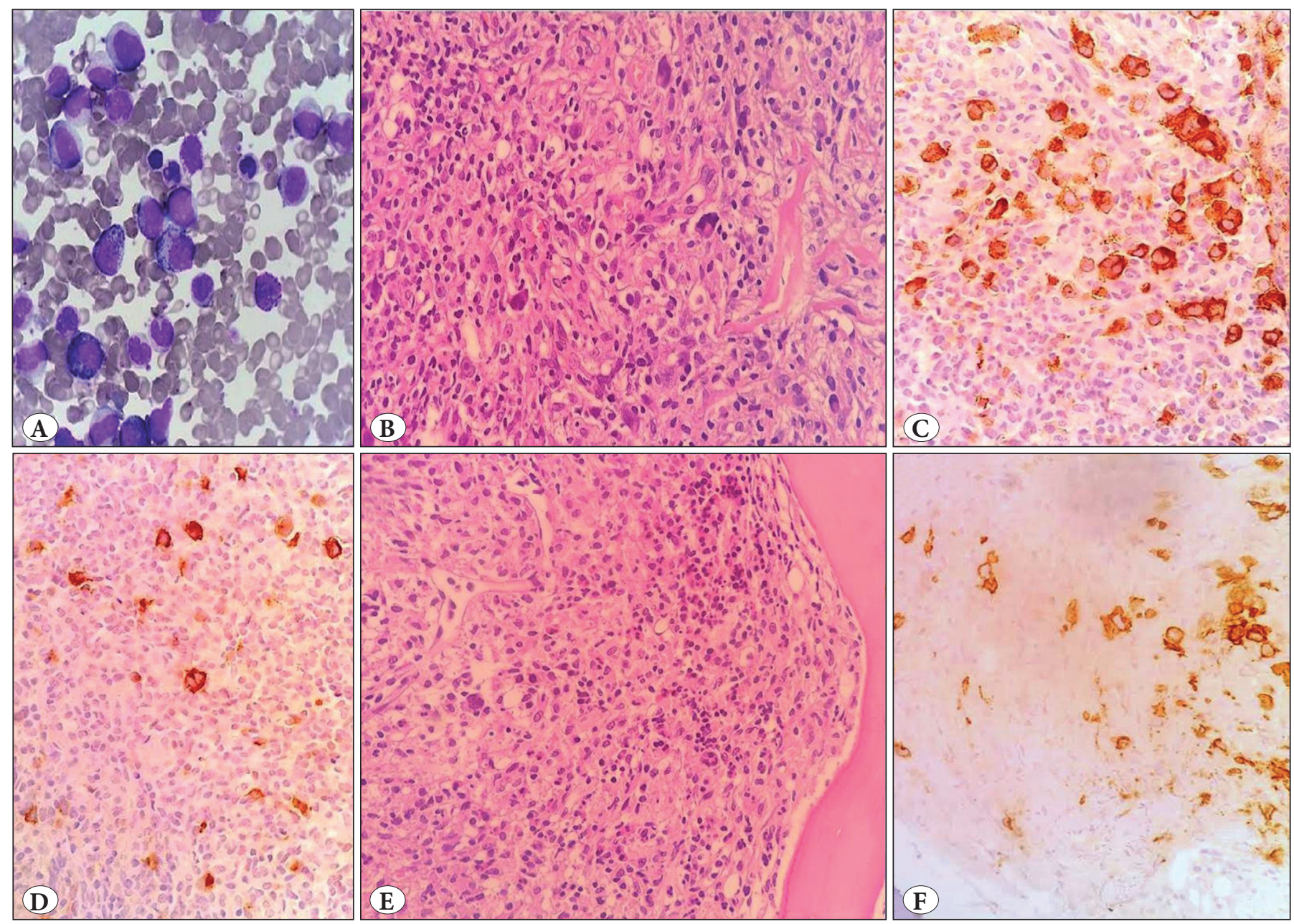

Figure 1: A) Peripheral blood smear showing hyperleucocytosis with neutrophil and myelocyte peak and basophilia (Wright -Giemsa; x200). B) Lymph node biopsy showing scattered large multilobulated Reed-Sternberg cells (H\&E; x40). C) Reed-Sternberg cells are positive for CD30 (CD30; x100). D) Reed-Sternberg cells are positive for CD15 (CD15; x100). E) Bone marrow biopsy showing infiltration by Reed-Sternberg cells (H\&E; x40). F) CD30 positive Reed-Sternberg cells (CD30; x100). 


\section{DISCUSSION}

Factors that are thought to contribute to the coexistence of hematological malignancies include host-specific characteristics, environment, history of previous chemotherapy and radiation or a possible common hematologic stem cell (4). Host-specific factors may include age, gender, race, genetic make-up, and life style choices. In the literature, there are multiple articles reviewing the association of prior treatment of chemotherapy or radiation and the development of second malignancy in the following months, particularly in Imatinib-treated CML. However, the incidence of secondary malignancies in CML patients is controversial as described in the literature. Miranda et al. analysed CML patients with secondary malignancies and found that 67 secondary malignancies developed in 64 out of 1525 CML patients in the chronic phase treated with TKI $(\mathrm{n}=61)$ and interferon- $\alpha$ only $(\mathrm{n}=3)$. The most common malignancies were prostate, colorectal and lung cancer, non-Hodgkin lymphoma (NHL), malignant melanoma, non-melanoma skin tumors and breast cancer (5). Roy et al. reported 6 cases of secondary malignancies among a French cohort of 189 CML patients treated with TKI (Imatinib) following Interferon failure for the first time in the literature in 2005. Out of six patients who developed a second malignancy, three patients developed prostate cancer and one patient each developed urinary bladder, colon and unknown site, with a duration of 33 to 110 months from CML diagnosis, and 8-36 months from the start of imatinib therapy. All these six patients had received an interferonbased regimen. The study suggested that the incidence of secondary malignancy among those patients and especially the incidence of prostate cancer was increased about four times compared to the incidence expected in the normal population (6). However, Pilot et al. published a data based on an epidemiological analysis performed by Novartis of second primary malignancies among 9518 patients in their global database treated with imatinib, which did not confirm the above observation. A second neoplasm was detected in 110 patients, but it was shown that the numbers of second cancers in clinical trials are similar to those expected based on the rates in the general population. (7). Verma et al. analysed patient cohorts from multiple phase I and II trials at the MD Anderson Cancer Center, who were treated with TKI for CML and other myeloproliferative neoplasms and showed that the standardized incidence ratio of second cancers was 0.60 , suggesting that the risk of secondary malignancies in patients treated with TKI was lower than expected in the general population, which was also in contrast to the observations by Roy et al, although the incidences of melanoma, endocrine tumors, kidney cancers, and CLL were higher than expected. The risk of secondary malignancies was lower than expected in the general population (8). Similarly, Helbig et al. found that a second malignancy developed in 8 patients (one case each of malignancy of endometrium, testis, skin melanoma, breast, bladder, prostate, large bowel and lung) out of 221 CML patients (3.6\%) treated with Imatinib with a median interval of 61 months (10-137 months). However, the risks for second malignancy development as well as for related death in CML patients were not statistically increased if compared to age-adjusted population (9). There was no case of Hodgkin lymphoma detected in the above studies. A few other studies demonstrated a higher incidence of secondary malignancy in CML patients. The incidence of second malignancy in CML patients in the post-imatinib era was significantly higher than the pre-Imatinib era (observed/ expected ratio of 1.48 versus 1.06) as demonstrated by Shah et al. by a survey done on the SEER (Surveillance, Epidemiology, and End Results) database. The highest risk of tumor development was found to be within 1 to 11 months after Imatinib initiation. The most frequent site of development of second malignancy was the digestive tract. Hodgkin lymphoma cases were not specified in the study (10). In a study done by Gunnarsson et al. that crosslinked the Swedish CML register to the Swedish Cancer registry, the risk of second malignancies was higher in the CML cohort from the imatinib era with a standardized incidence ratio of 1.52 and a median follow-up of 3.7 years (range $0-9.9$ years) (11). In the Czech Republic and Slovakia, a retrospective analysis of $1038 \mathrm{CML}$ patients treated with tyrosine kinase inhibitors between 2000 and 2009 was conducted by Voglova et al. A second malignancy was detected in 35 patients (3.37\%) with median interval of 32 months from the initiation of tyrosine kinase inhibitor therapy. The study found that the age-standardized incidence rate of all malignant tumors (except non melanoma skin cancers) was 1.5 fold higher in CML patients treated with TKI than in the normal population (6.76/1,000 person-years vs. $9.84 / 1,000$ person-years), although the difference was statistically insignificant (12). The very rare occurrence of concurrent Hodgkin disease in a CML patient was reported by Sharkunov et al. (13).

The pathophysiological or molecular mechanism of development of Hodgkin lymphoma in CML patients treated with Imatinib is largely unknown. However, CML itself is a risk factor for development of solid cancers and hematologic malignancies as the acquired BCR-ABL translocation at the time of CML diagnosis and additional 
chromosomal abberations as a sign of clonal evolution during the course of disease show the potential of genetic instability in CML. Therefore, progenitors may already have the capacity to enforce themselves as distinct cells with enhanced malignancy resulting in solid cancers or hematologic malignancies before or later in CML (14). In addition, Imatinib has an immunoregulatory effect by inhibiting T-cell activation and proliferation as well as by diminishing the capacity of dendritic cells to elicit primary T-cell responses (15). The exposure to Imatinib induces centrosome and chromosome aberrations in cultures of normal human dermal fibroblasts, Chinese hamster embryonal and Indian muntjac fibroblasts in a significant, dose-dependent and species-independent manner. Those aberrant karyotypes emerging under Imatinib use were irreversible after a prolonged culture omitting the drug. Thus, these observations suggest that neoplastic, chromosomally unstable clones may be developed de novo from normal non-hematopoietic cells by Imatinib (16). Genetic instability caused by centrosome defects has an important influence in early steps of the development as well as in the progression of many cancers (17-19). In addition, the c-Abl tyrosine kinase was found to promote DNA damage-induced apoptosis. The inhibition of apoptosis associated by TKIs may also explain a proliferative potential of those drugs (20). EBV has been etiologically associated with a proportion of classical HL patients. EBV latent membrane protein (LMP)-1 and/or EBV-encoded small RNAs (EBERs) have been detected in $40 \%$ of classical HL patients in economically developed countries (21). Patients on chemotherapy are immunocompromised and may be at greater risk of neoplasm driven by infectious agents such as EBV and Hodgkin lymphoma.

Lymphadenopathy in a known case of CML may be due to extra-medullary blast crisis or development of second malignancy de novo or due to previous chemotherapy. A correct diagnosis requires histopathology with immunohistochemistry and molecular analysis. As Hodgkin lymphoma in a known case of CML is very rare, further studies are also needed to know the pathogenic relationship between the two entities and to assess the risk of Hodgkin lymphoma in CML patients treated with TKI.

\section{CONFLICT OF INTERESTS}

The authors declared no conflict of interest.

\section{REFERENCES}

1. Sattler M, Griffin JD, Mechanisms of transformation by BCR/ ABL oncogene. Int J Hematol. 2001;73:278-91.

2. Gambacorti-Passerini C, Antolini L, Mahon FX,et al. Multicenter independent assessment of outcomes in chronic myeloid leukemia patients treated with imatinib. J Natl Cancer Inst. 2011; 103: 553-61.

3. Rebora P, Czene K, Antolini L, Passerini CG, Reilly M, Valsecchi MG. Are chronic myeloid leukemia patients more at risk for second malignancies. A population-based study. Am J Epidemiol. 2010; 172: 1028-33

4. Maerki J, Katava G, Siegel D, Silberberg J, Bhattacharyya PK. Unusual case of simultaneous presentation of plasma cell myeloma, chronic myelogenous leukemia, and a jak2 positive myeloproliferative disorder. Case Rep Hematol. 2014;2014:738428.

5. Miranda MB, Lauseker M, Kraus MP, Proetel U, Hanfstein B, Fabarius A, Baerlocher GM, Heim D, Hossfeld DK, Kolb HJ, Krause SW, Nerl C, Brümmendorf TH, Verbeek W, Fauser AA, Prümmer O, Neben K, Hess U, Mahlberg R, Plöger C, Flasshove M, Rendenbach B, Hofmann WK, Müller MC, Pfirrmann M, Hochhaus A, Hasford J, Hehlmann R, Saußele S. Secondary malignancies in chronic myeloid leukemia patients after imatinib-based treatment: Long-term observation in CML Study IV. Leukemia. 2016; 30:1255-62.

6. Roy L, Guilhot J, Martineau G, et al. Unexpected occurrence of second malignancies in patients treated with interferon followed by imatinib mesylate for chronic myelogenous leukemia. Leukemia. 2005;19:1689-92.

7. Pilot PR, Sablinska K, Owen S, Hatfield A. Epidemiological analysis of second primary malignancies in more than 9500 patients treated with imatinib. Leukemia. 2006;20:148.

8. Verma D, Kantarjian H, Strom SS, Rios MB, Jabbour E, QuintasCardama A, Verstovsek S, Ravandi F, O'Brien S, Cortes J. Malignancies occurring during therapy with tyrosine kinase inhibitors (TKIs) for chronic myeloid leukemia (CML) and other hematologic malignancies. Blood. 2011;118:4353-8.

9. Helbig G, Bober G, Seweryn M, Wichary R, Tukiendorf A, Sedlak L, Oleksy T, Kyrcz-Krzemień S. Occurrence of secondary malignancies in chronic myeloid leukemia during therapy with imatinib mesylate-single institution experience. Mediterr J Hematol Infect Dis. 2015;7:e2015003.

10. Shah BK, Ghimire KB. Second primary malignancies in chronic myeloid leukemia. Indian J Hematol Blood Transfus. 2014;30:236-40

11. Gunnarsson N, Stenke L, Höglund M, Sandin F, Björkholm M, Dreimane A, Lambe M, Markevärn B, Olsson-Strömberg U, Richter J, Wadenvik H, Wallvik J, Själander A. Second malignancies following treatment of chronic myeloid leukaemia in the tyrosine kinase inhibitor era. Br J Haematol. 2015; 169:6838 
12. Voglova J, Muzik J, Faber E, Zaclova D, Klamova H, Steinerova K, Michalovicova Z, Demitrovicova L, Cmunt E, Novakova L, Tothova E, Belohlavkova P, Mayer J, Indrak K. Incidence of second malignancies during treatment of chronic myeloid leukemia with tyrosine kinase inhibitors in the Czech Republic and Slovakia. Neoplasma. 2011;58:256-62.

13. Sharkunov NN, Moiseyeva TN, Zybunova EE, Vinogradova OY, Kravchenko SK. Successful treatment for Hodgkin's lymphoma in a female patient with $\mathrm{Ph}+$ chronic myeloid leukemia. Ter Arkh. 2012;84:71-4.

14. Fabarius A, Kalmanti L, Dietz CT, Lauseker M, Rinaldetti S, Haferlach C, Göhring G, Schlegelberger B, Jotterand M, Hanfstein B, Seifarth W, Hänel M, Köhne CH, Lindemann HW, Berdel WE, Staib P, Müller MC, Proetel U, Balleisen L, Goebeler ME, Dengler J, Falge C, Kanz L, Burchert A, Kneba M, Stegelmann F, Pfreundschuh M, Waller CF, Spiekermann K, Brümmendorf TH, Edinger M, Hofmann WK, Pfirrmann M, Hasford J, Krause S, Hochhaus A, Saußele S, Hehlmann R; SAKK and the German CML Study Group. Impact of unbalanced minor route versus major route karyotypes at diagnosis on prognosis of CML. Ann Hematol. 2015;94: 2015-24.

15. Appel S, Rupf A, Weck MM, Schoor O, Brummendorf TH, Weinschenk T, Grünebach F, Brossart P. Effects of imatinib on monocyte-derived dendritic cells are mediated by inhibition of nuclear factor-kappa B and Akt signaling pathways. Clin Cancer Res. 2005; 11: 1928-40.
16. Fabarius A, Giehl M, Frank O, Duesberg P, Hochhaus A, Hehlmann R, Seifarth W. Induction of centrosome and chromosome aberrations by imatinib in vitro. Leukemia. 2005; 19: $1573-78$.

17. Pihan GA, Purohit A, Wallace J, Malhotra R, Liotta L, Doxsey SJ. Centrosome defects can account for cellular and genetic changes that characterize prostate cancer progression. Cancer Res. 2001; 61:2212-19.

18. Pihan GA, Wallace J, Zhou Y, Doxsey SJ. Centrosome abnormalities and chromosome instability occur together in preinvasive carcinomas. Cancer Res. 2003; 63: 1398-404.

19. Mayer F, Stoop H, Sen S, Bokemeyer C, Oosterhuis JW, Looijenga LHJ. Aneuploidy of human testicular germ cell tumors is associated with amplification of centrosomes. Oncogene. 2003; 22:3859-66.

20. Yuan ZM, Huang Y, Ishiko T, Kharbanda S, Weichselbaum R, Kufe D. Regulation of DNA damage-induced apoptosis by the cAbl tyrosine kinase. Proc Natl Acad Sci USA. 1997; 94:1437- 40.

21. Jarrett RF, Krajewski AS, Angus B, Freeland J, Taylor PR, Taylor GM, Alexander FE. The Scotland and Newcastle epidemiological study of Hodgkin's disease: Impact of histopathological review and EBV status on incidence estimates. J Clin Pathol. 2003;56:8116. Erratum in: J Clin Pathol. 2004; 57:112. 